\title{
ON THE HYPER-GEOMETRIZATION OF RELATIVISTIC PHASE-SPACE FORMALISM. II
}

\author{
By \\ J. I. HoRváth \\ DePartment of Theoretical Physics, József atTila university, szeged
}

(Presented by A. Kónya. - Received 30. V. 1967)

In the first part of this paper it was emphasized that in the framework of line-element geometry - via the dependence of the dynamic quantities on directions in space-time internal dynamic relations of the considered system can be geometrized. For the sake of simplicity this idea of hyper-geometrization was discussed in the case of a pseudo-Euclidean space-time continuum. In this part, the internal dynamic degrees of freedom, their symmetries and phasespace picture will be treated generally and reformulated for inhomogeneous and anisotropic dynamic systems, not only in terms of the tetrad formalism of Riemannian geometry but also on the basis of the direct generalization of the suggested triad formalism. Finally, a new general definition of the relativistic phase-space volume and its relation to previous suggestions will be discussed, with some applications in the theory of fermion gases.

In Part I [28] we dealt in detail with the formulation of the general idea of our suggested geometrization of the relativistic phase-space formalism in the pseudo-Euclidean space-time continuum. To make it possible to obtain a deeper insight into the real geometrical structure of the relativistic phasespace it seems to be worth while to generalize the suggested method in spite of the fact that from the points of view of several applications of the theory the suggested formalism would be general enough. In fact, having in mind the framework of line-element geometry and the definition of inhomogeneous direction co-ordinates, respectively, the development of the general formalism does not mean any considerable trouble, and based on the general theory, the various applications can be obtained by fairly simple specialization.

In order to reformulate the idea of hyper-geometrization quite generally it seems to be worth-while to keep in mind a general method for the geometrization of external fields suggested previously $[31]^{*}$ and the tetrad-formalism of Riemannian geometry. It will be shown that in terms of the suggested method a natural general definition of the phase-space volume can be proposed which is a direct genc ralization of the well-known non-relativistic formalism. As an application, the calculation of the $z \in$ ro point kint tic energy of perfect fermion gases in Riemannian space-time continuum will be reviewed.

* Numeration of paragraphs, formulae and references will be continued in this Part and, of course, our notations are co-ordinated with those of Part I [28]. 


\section{\$ 3. Relativistic phase-space in general spaces}

The definition of the local momentum space in general (most often Riemannian) spaces does not need any essentially new idea. Of course, one has to take into account that the normalization condition of the four-momenta, (i.e., the equation of the mass shell) instead of eq. (2.2.1) is, in the case of gas particles with rest-mass $m_{0}$,

$$
g_{\mu \nu}(x) p^{\mu} p^{v}=m_{0}^{2},
$$

where $g_{\mu v}(x)$ denotes the metric fundamental tensor of the space considered. This means, however, that, e.g., for the time component of the momenta the expression

$$
p_{0}= \pm\left\{m_{0}^{2} g_{00}+\left(g_{0 i} g_{0 k}-g_{00} g_{i k}\right) p^{i} p^{k\}^{1 / 2}}\right.
$$

can be obtained.

In particular, bearing in mind the special case of zero rest-mass particles $\left(m_{0}=0\right)$, i.e., light-like four-momenta, we have

and

$$
g_{\mu \nu}(x) p^{\mu} p^{\nu}=0
$$

$$
p_{0}= \pm\left\{\left(g_{0 i} g_{0 k}-g_{00} g_{i n}\right) p^{i} p^{k}\right\}^{1 / 2}
$$

respectively. We shall meet such special cases when the calculation of the zeropoint kinetic energy of neutrino gas will be treated in Section 4.6. It will be shown that those problems connected with the zero rest-mass of the gas particles by the limiting process $m_{0} \rightarrow 0$ can be mastered. As a matter of fact, without loss of generality it can be supposed in the following that $m_{0} \neq 0$.

In order to carry out the generalization of the suggested hyper-geometrization the mean problems to be solved are the orientation of the $\lambda$-triads and the definitions of the group of internal transformations, respectively, which will be discussed after some preparation.

3.1. General method for the geometrization of external fields. In the framework of Einstein's theory of gravitation the equations of motion of test particles are given by the equations of the geodetic lines of the suitable Riemannian space:

$$
\frac{d^{2} x^{\mu}}{d \sigma^{2}}+\left\{\begin{array}{c}
\mu \\
x \cdot \lambda
\end{array}\right\} \frac{d x^{\varkappa}}{d \sigma} \frac{d x^{\lambda}}{d \sigma}=0
$$

where $\left\{\begin{array}{c}\mu \\ x \cdot \lambda\end{array}\right\} x^{\mu}=x^{\mu}(\sigma)$ and $\sigma$ denote the Christoffel's symbols, the equations of the worldline of the particles considered and the Riemannian length of arc, respectively.

Switching on an external field of force $K^{\mu}$ the equations of motion are

$$
\frac{d^{2} x^{\mu}}{d \sigma^{2}}+\left\{\begin{array}{c}
\mu \\
x \cdot \lambda
\end{array}\right\} \frac{d x^{\varkappa}}{d \sigma} \frac{d x^{\varkappa}}{d \sigma}=K^{\mu}
$$


Keeping Nelli's generalization of the Riemannian geometry in mind, the geometrization of such an external field of force can be carried out - without changing the original Riemannian metric - by the introduction of new parameters of affine connection [29-31]. Namely, it can be shown that replacing the Christoffel's symbols $\left\{\begin{array}{c}\mu \cdot \lambda\} \\ \varkappa \cdot \lambda\end{array}\right\}$ the new parameters of affine connections

$$
L_{\varkappa \mu \lambda} \stackrel{\operatorname{def}}{\{x \cdot \lambda\}}\left\{\begin{array}{c}
\mu \\
x \cdot \lambda \mu \lambda
\end{array}\right.
$$

having the same transformation laws as those of the Christoffel's symbols, where $1_{\chi \mu \lambda}$ mean the components of an arbitrary tensor being antisymmetric in their first two indices, i. e.

$$
A_{\mu x \lambda}=-A_{\kappa \mu \lambda}
$$

the metrical properties (e. g., geodesics, invariant differential of $g_{\mu \nu}$ etc.) of the original Riemannian space do not change, but its geometrical structure will be generalized. This generalization of the geometrical structure - in spite of the unchanged metrical structure - results in the considerable fact that the geodetic lines of the space and the autoparallel curves of the geometry will be different.

Based on an adequate connection between the introduced antisymmetric tensor and the field of force $K^{\mu}$, the suggested geometrization of the external field of force means that the test particles are moving without external field along geodetic lines and under the influence of external field of force along autoparallel curves, respectively.

The equations of the autoparallel curves are

$$
\frac{d^{2} x^{\mu}}{d \sigma^{2}}+L \underset{\varkappa \cdot \lambda}{\mu} \frac{d x^{\varkappa}}{d \sigma} \frac{d x^{\lambda}}{d \sigma}=0
$$

which, owing to the definition (3.1.3) of $L_{\mu \times \lambda}$. can be put in the form

$$
\frac{d^{2} x^{\mu}}{d \sigma^{2}}+\left\{\begin{array}{c}
\mu \\
\varkappa \cdot \lambda
\end{array}\right\} \frac{d x^{\varkappa}}{d \sigma} \frac{d x^{\lambda}}{d \sigma}=-\Lambda \underset{x \cdot \lambda}{\mu} \frac{d x^{\varkappa}}{d \sigma} \frac{d x^{\lambda}}{d \sigma} .
$$

E. $g .$, in the case of an electromagnetic field with the field tensor $F_{x \mu}$ owing to the familiar definition

$$
j^{\lambda}=e \frac{d x^{\lambda}}{d \sigma}
$$

of the current corresponding to a moving point-particle with the electric charge $e$, let us suppose that

$$
A_{\mu x \lambda}=\boldsymbol{F}_{x \mu} j \lambda
$$

having the symmetry property required by the definition (3.1.4) of $1_{x \mu \lambda}$. This means, however, that

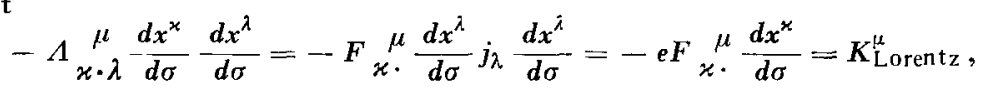

being just the components of the well-known force of Lorentz.

Bearing this method of geometrization of external fields in mind, fairly general homogeneous gas systems under the influence of external fields can be treated on the bases of the framework of geometrization of the phase-space suggested in the following. Indeed, let us consider a system of gas particles being in a gravitation and also in some kind of external field. Then the influence of gravitation can be mastered on the basis of Einstein's theory of gravitation via the metrical fundamental tensor $g_{\mu \nu}(x)$ of the suitable Riemannian space and of the other external field in terms of the method reviewed above. If also the gravitational interaction of the particles have to be taken into account, this can in principle be done - as was pointed out by ISRAEL [9] - via a self-consistent background field.

As a matter of fact, when for the sake of simplicity a Riemannian space-time background is mentioned below, this is not to be regarded as an essential loss of generality, because the mentioned generalization can automatically be included. However, it must be emphasized that in the kinetic theory of gases - even in the case of perfect gas systems the interactions of the particles via elastic binary collisions play an important part. Therefore, this kind of interaction has to be treated separately below (Section 5.2). 


\subsection{Definition of the internal co-ordinates in Riemannian space-time. Owing} to the remarks at the end of Section 3.1., it may be supposed without any essential restrictions that the history of the assemblage is to be conceived as a network of time-like world-lines in a Riemannian space-time.

Having in mind the definition of the inhomogeneous direction co-ordinates of the three-dimensional local momentum-space, let us introduce in every point of the space-time continuum the $\lambda$-triad formed by three mutually orthogonal vectors $\lambda_{i}^{\mu}(i=1,2,3)$ of unit length which are orthogonal to the time-like four-momentum $p_{(0)}^{v}$ of the gaseous particles in the local rest frame of reference $K^{0}$. Owing to the Riemannian space-time metric, and the condition of orthonormality and the definition of the inhomogeneous direction co-ordinates, i.e. eqs. $(2.4 .2)-(2.4 .5)$ have to be replaced by

$$
\begin{aligned}
& g_{\mu \nu}(x) \underset{i}{\lambda_{j}^{\mu}} \lambda^{\nu}=\eta \delta_{i j}, \\
& g_{(0) \mu \nu}(x) \underset{i}{\lambda_{(0)}^{\mu}} p_{(0)}^{v}=0, \\
& \vartheta_{i} \stackrel{\text { def }}{=} \operatorname{arc} \cos \left\{g_{\mu v}(x) \underset{i}{\lambda^{\mu}} p^{\nu} / m_{0}\right\} \\
& \xi_{i} \stackrel{\text { dei }}{=} \cos \vartheta_{i} \equiv g_{\mu \nu}(x) \underset{i}{\lambda_{i}^{\mu}} p^{\nu} / m_{0}
\end{aligned}
$$

Of course, the new inhomogeneous direction co-ordinates also are unambiguously determined up to the sign of $p^{0}$.

In order to settle the orientation of the $\lambda$-triad at any point or at different points of the space-time continuum it seems to be simple to use the framework of the orthonormal tetrad formalism of the Riemannian spaces discussed in detail by SYNGE [32] as especially favourable for our purposes.

As an orthonormal tetrad, four unit vectors $A_{(\alpha)}^{\mu}$ orthogonal by pairs are denoted where the indices in parentheses likes $(\alpha)$ indicate a label distinguishing the particular axes.

The covariant components of the same tetrad are

$$
A_{(\mathrm{x}) \mu}=g_{\mu \lambda} A_{(\alpha)}^{10}
$$

Three of the axes are, of course, space-like and one is time-like. We shall always so label the axes that $\Lambda_{(1)}^{\mu}$ is time-like.

The conditions of orthonormality can be written in the form:

where

$$
\Lambda_{(\alpha)}^{\mu} \Lambda_{(\beta) \mu}=\eta_{(\alpha \beta)},
$$

$$
\begin{gathered}
\eta_{(00)}=-\eta_{(11)}=-\eta_{(22)}=-\eta_{(33)}=1, \eta_{(\alpha \beta)}=0(\alpha \neq \beta) ; \\
\eta_{(\alpha \beta)}=\eta^{(\alpha \beta)}
\end{gathered}
$$

is a diagonal matrix; it satisfies the relation

$$
\eta^{(\alpha \beta)} \eta_{(\beta \gamma)}=\delta_{\gamma}^{x}
$$

being, in the language of matrix algebra, a square root of unity. 
One has to emphasize that the labels on the vectors have no tensorial meaning; nevertheless, by means of the $\eta$-matrix the framework of the tensor calculus can be introduced. Let the raising and lowering of the labels be defined by

$$
\Lambda^{(\alpha) \mu}=\eta^{(\alpha \beta)} \Lambda_{(\beta)}^{\mu} \quad \text { and } \quad \Lambda_{\mu}^{(\alpha)}=\eta^{(\alpha \beta)} \Lambda_{(\beta) \mu},
$$

then owing to eqs. (3.2.8) we have

$$
A_{(\alpha)}^{\mu}=\eta_{(\alpha \beta)} A^{(\beta) \mu} \quad \text { and } \quad A_{(\alpha)_{\mu}}=\eta_{(\alpha \beta)} A_{\mu}^{(\beta)}
$$

respectively. Finally, the relations

$$
\Lambda_{(\alpha)}^{\mu} \Lambda_{\mu}^{(\beta)}=\delta_{x}^{\beta} \quad \text { and } \quad \Lambda_{(\alpha)}^{\mu} \Lambda_{\nu}^{(\alpha)}=\delta_{\nu}^{\mu}
$$

can be obtained. The two tetrads $A_{(x)}^{\mu}$ and $\Lambda^{(\alpha) \mu}$ are closely connected: their space-like axes are the same and their time-like ones are opposed to one another, $\dot{x}_{\text {. }}$ e., they differ in their handedness.

Let us give at a space-time point two orthonormal tetrads, $\Lambda_{(x)}^{\mu}$ and $\mathcal{A}_{(\alpha)}^{\prime \mu}$ that can be connected by a Lorentz transformation with the so-called Lorentz matrix

$$
L_{(\alpha),--}^{(\alpha), ~ d e i} A_{\mu}^{(\alpha)} A_{(\alpha)}^{\prime \mu}
$$

being the unit matrix if the two tetrads coincide. Owing to eqs. (3.2.11) and (3.2.12) at every space-time point the equivalent Lorentz transformations:

$$
\Lambda_{(\alpha)}^{\prime \mu}=L_{(\alpha)}^{(\alpha)}, \Lambda_{(\alpha)}^{\mu} \text { and } \Lambda_{\mu}^{(\alpha)}=L_{(\beta)}^{(\alpha)}, \Lambda_{p^{\prime}}^{\prime(\beta)}
$$

can be introduced, being independent of any changes of the space-time coordinates. These Lorentz transformations may be interpreted as the "internal" changes of the orientation of the tetrads.

Now, let us suppose that in the local rest frame of reference $K^{0}$ of the gaseous particles, distinguished by the original tetrad $A_{(x)}^{* \mu}$, we have

$$
p_{(0)}^{\mu} \stackrel{\text { def }}{=} A_{(0)}^{* \mu}, \lambda_{i}^{\mu} \stackrel{\text { dei }}{=} A_{(i)}^{* \mu} \quad(i=1,2,3)
$$

then one can immediately see that the $\lambda^{+}$-triad as the space-like part of the Lorentz-covariant $\Lambda_{(\alpha)}^{* \mu}$ tetrad and the $\lambda^{-}$-triad as the space-like part of the Lorentz-contravariant $-A^{*(\alpha) \mu}$ tetrad has to be defined.

As a matter of fact, any changes of the orientation of the $\lambda$-triad generating the group of internal transformations - which owing to the new definition (3.2.4) of the inhomogeneous direction co-ordinates will be denoted by $\varphi_{f \xi}-$ is the spatial subgroup of the invariant Lorentz transformations (3.2.12).

3.3. Orientation of the local frames of reference and the invariant momentum space. Associated with each point of a curve $x^{\mu}=x^{\mu}(\sigma)$ in space-time an orthonormal tetrad can be introduced with particular considerable features formed by the unit tangent

$$
t_{(0)}^{\mu} \stackrel{\text { der }}{\frac{d x^{\mu}}{d \sigma}}
$$


as well as by the first, second and third normals to the curve denoted by $n_{(1)}^{\prime \prime}$, $\boldsymbol{n}_{(2)}^{\mu}$ and $\boldsymbol{n}_{(3)}^{\mu}$, respectively. These orthogonal by pairs unit vectors are determined by means of the well-known Frenet-Serret formulae:

$$
\begin{array}{ll}
\frac{D}{d \sigma} t_{(0)}^{\mu} & =\varrho_{1} n_{(1)}^{\mu}, \\
\frac{D}{d \sigma} n_{(0)}^{\mu} & =\varrho_{2} n_{(2)}^{\mu}+\varrho_{1} t_{(0)}^{\mu}, \frac{D}{d \sigma} n_{(2)}^{\mu}=\varrho_{3} n_{(3)}^{\mu}-\varrho_{2} n_{(1)}^{\mu},
\end{array}
$$

where the scalars $\varrho_{1}, \varrho_{2}$ and $\varrho_{3}$ are the first, second and third curvatures of the curve considered. In the case of time-like curves, i.e., in the case of curves with time-like unit tangents, we have:

$$
g_{\mu \nu} t_{(0)}^{\nu} t_{(0)}^{v}=1, g_{\mu \nu} n_{(i)}^{i} n_{(i)}^{v}=-1 \quad(i=1,2,3)
$$

This so-called normal tetrad $\left\{t_{(0)}^{\prime \prime}, n_{(i)}^{\mu}\right\}$ to the curves will be used below when the orientation of the tetrads at different distinct space-time points are compared.

When considering, at any two distinct points of the Riemannian spacetime continuum, a tetrad and $\lambda$-triad, their orientation has to be compared by means of the framework of the general parallel transport along the world lines of the particles.

The world lines of the particles are time-like curves with equations $x^{\mu}=x^{\prime \prime}(\sigma)$. It is well-known that a vector $V^{\mu}$ is said to undergo parallel transport along a curve if its absolute derivative vanishes

$$
\frac{D V^{\prime \mu}}{d \sigma}-\frac{d V^{\mu}}{d \sigma}+\left\{\begin{array}{c}
\mu \\
\kappa \cdot \lambda
\end{array}\right\} V^{\text {def }} \times \frac{d x^{\lambda}}{d \sigma}=0 .
$$

In the following it seems to be more favourable to use a particular kind of parallel transport of a vector $V^{\mu}$ - called usually a FERMI-WALKER transport $[32,33]$ - along the world lines of the particles defined by the equation

$$
\frac{D V^{\mu}}{d \sigma}=\frac{\varrho_{1}}{m_{0}} V_{\varkappa}\left(p^{\mu} n_{(1)}^{\varkappa}-p_{(1)}^{\varkappa}\right),
$$

where attention has been paid to the fact that in the case of particles world lines $x^{\mu}=x^{\mu}(\sigma)$ the unit tangent is precisely the four-momentum of the particles normalized to unity:

$$
t_{(0)}^{\mu}=\frac{d x^{\mu}}{d \sigma}=p^{\mu} / m
$$

As important features of the FERMI-WALKer transport are that 
(1) the unit tangent $t_{(0)}^{\mu}$ itself automatically undergoes FERMI - WALKER transport, as can be checked on the basis of eqs. (3.3.5) and (3.3.2) immediately;

(2) it resembles parallel transport in the conservation of magnitu de and scalar product;

(3) if the FERMI-WaLker transport is applied to the normals $\eta_{(i}^{u}$ which are orthogonal to the tangent $t_{(0)}^{\mu}$ at some point of the curve considered it remains, of course, orthogonal to $t_{(0)}^{\mu}$ and to each other. This means, however, that the normal tetrad $\left\{t_{(0)}^{\mu}, n_{(i)}^{\mu}\right\}$ under FERMI-WALKER transport remains a normal tetrad along any curves.

As a matter of fact, the comparison of the orientations of two orthonormal tetrads at two distinct points $\left\{x^{\mu}\right\}$ and $\left\{y^{\mu}\right\}$ of the Riemannian spacetime can be mastered in the following way;

Let the two considered points be connected by a world-line with a unit tangent the direction of which, e.g. at the point $\left\{x^{\mu}\right\}$, coincides with that of the $t_{(0)}^{\mu}$ axis of the tetrad considered. Then, let the orthonormal tetrad with its origin, e.g. at $\left\{x^{\mu}\right\}$, be subjected to FERMI - WALKER transport until its origin coincides with $\left\{y^{\mu}\right\}$. In this way a virtual tetrad is unambiguously oriented which can be used as a basis to determine the orientation of the second tetrad with its origin originally at $\left\{y^{\mu}\right\}$, by means of the method for the comparison of tetrads at the same space-time point in terms of the internal Lorentz transformations.

The comparison of the $\lambda$-triads of different space-time points, having their definition in mind, based on that of the tetrads is straightforward and need not be gone into. However, then the inhomogeneous direction co-ordinates $\left\{\xi_{i}\right\}$ of the local momentum-spaces originally defined at different spacetime points can be "synchronized" and the relativistic phase-space, also in Riemannian space-times, can be defined as a direct product of the configuration spaces and momentum spaces, respectively.

Finally, we have to mention that the definition of the inhomogeneous direction co-ordinates - the framework of which, having the invariant characterization of the internal degrees of freedom of physical fields in mind, was suggested several years ago [16] - seems to be very close to that of the spatial set of Fermi co-ordinates [32] the advantages of which from other points of view were emphasized by Synge [32].

3.4. Geometrization of the relativistic phase-space of dynamically anisotropic gas systems. Bearing the framework of Section 2.5. in mind, if some dynamical anisotropy of the considered gas system exists characterised by the metrical fundamental tensor $g_{\mu \nu}(x, p)$ of the suitable line-element space, the conditions of orthonormality (3.2.1)-(3.2.2) and the definition of the inhomogeneous direction co-ordinates $(3.2 .4)$ have to be replaced by the fairly general equations

$$
g_{\mu \nu}(x, p) \underset{i}{\lambda^{\mu}} \underset{k}{\lambda^{v}}=\eta \delta_{i k}
$$




$$
\left.g_{(0) \mu \nu}(x, p){\underset{i}{(0)}}_{\lambda^{u}} p_{(0)}^{y}=0, \text { (in the rest system } K^{\circ}\right)
$$

and

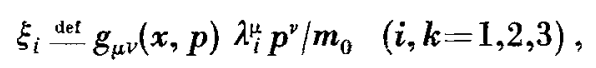

respectively; of course, with the normalization of the four-momenta, i.e., the equation of the mass-shell:

$$
g_{\mu \nu}(x, p) p^{\mu} p^{\nu}=m_{0}^{2}
$$

corresponding to the general definition (2.3.5) of the unit vector

$$
l^{\mu} \text { def } v^{\mu} / F=p^{\mu} / m_{0}
$$

which have the same direction as that of the line-element $(x, v)$. The tildes over the symbols $\lambda^{\mu}, p^{\mu}$ and $\xi_{i}$ - introduced previously in the analogous case at the end of Section 2.5 - for the sake of a simplified notation here and in the following may be omitted without any risk of confusion if we agree that the metrical properties of the vectors always have to be understood in terms of the particular metrical fundamental tensor actually considered.

Taking the framework of the line-element geometry, i.e. the new definition of the metrical fundamental tensor into account, the orientation of the $\lambda$-triad and the general definition of the invariant momentum space can be formulated, essentially in the same way as has been discussed in the previous Section 3.3.

However, let us emphasize that in the case of a special but, from the point of view of applications in physics, very large class of general line-element spaces, the orientation of the a-triads can be mastered in a more straightforward way:

Let us suppose that in the general line-element space considered a field of directions $\lambda^{\prime \prime}=v^{\mu}(x)$ exists satisfying the differential equations

where

$$
\frac{d i^{\mu}}{d x^{\varrho}}+\widetilde{\Gamma}_{*}^{\mu} \stackrel{\mu}{\mu}=0
$$

and

with

$$
\frac{d l^{\mu}}{d x^{\varrho}}=\mathrm{a}_{\varrho} l^{\mu \stackrel{\text { def }}{=}} \frac{\partial}{\partial x^{\ell}} l^{\prime \prime}(x, v(x))
$$

$$
\widetilde{T}_{x \cdot \tau}^{\alpha} \stackrel{\text { def }}{=} \Gamma_{x \cdot \tau}^{\alpha}-A_{x \cdot \lambda}^{\alpha} \Gamma_{* \cdot \tau}^{\lambda}
$$

$$
A_{x \mu \lambda} \stackrel{\text { def }}{=}-\frac{1}{2} \frac{\partial g_{x \mu}}{\partial p^{\lambda}}
$$

the latter being the so-called torsion tensor of the linerelement space. Finally, the abbreviation

$$
\Gamma_{* \cdot \tau}^{\lambda} \stackrel{\text { det }}{=} \Gamma_{\nu \cdot \tau}^{\lambda} p^{\nu}
$$

is introduced; i. e., an asterisk instead of a covariant or contravariant index means contraction with the unit vector $l^{\mu}$. 
The condition of integrability of the differential equations (3.4.6) is

$$
\partial_{\varrho} \partial_{\tau} l^{\mu}-\partial_{\tau} \partial_{\varrho} l^{\mu} \stackrel{\text { def }}{=}-\widetilde{R} \underset{*}{\mu} \stackrel{\mu}{\mu}=0
$$

where

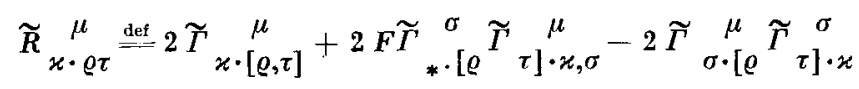

is the tensor of principal curvature of the line-element space. Here the following abbreviations have been used: Let $T_{\mu \nu}$ be an arbitrary tensor, then

and

$$
T_{\mu \nu, \varrho}=\partial_{\varrho} T_{\mu \nu}
$$

$$
T_{[\mu \mid \tau \nu \nu]} \stackrel{\text { det }}{=} \frac{1}{2}\left\{T_{\mu \tau \nu}-T_{\nu \tau \mu}\right\}
$$

denotes the Schouten's commutator.

This means, however, that our line-element space is "quasi-flat"; i. e., strictly speaking, in our space of line-elements absolute parallelism of the line-elements exists. However, one has to be aware that the absolute parallelism of the line-elements is an essentially different concept from the absolute parallelism of vectors, the latter being a characteristic property of Euclidean point-spaces.

The absolute parallelism of the line-eiements only means that for any given direction at an arbitrary space-time point a parallel direction in the sense of Levi-Civita can unambiguously be determined at all other points of the space-time continuum. But just this is needed if the orientation of the $\lambda$-triads is to be carried out.

As matters stand, two methods are at our disposal for the orientation of the basic $\lambda$-triads of the local momentum space. From the intuitive point of view of applications the second method, based on the concept of the absolute parallelism of the line-elements, has some advantage being a direct generalisation of the familiar framework accustomed to in the case of pseudo-Euclidean spaces, discussed in the previous paragraph in detail.

\section{\$ 4. General definition of the relativistic phase-space volume}

Previous definitions of the relativistic phase-space volume have usually been proposed either in the language of special co-ordinate systems or based on invariance in respect to a group of transformations properly introduced. If the suggested framework of the general line-element geometry could be accepted, it has to be used, of course, the concept of the hyper-surfaces of the line-element spaces is more complicated than that of the usual point-geometries. In fact, the complications arise from the dependence of the geometrical quantities and relations on the directions as well. Strictly speaking, a set of so-called osculate Riemannian spaces belongs to all points $\left\{x^{\mu}\right\}$ of the co-ordinate- (configuration-) space and the line-element space can be regarded as the ensemble of osculate Riemannian spaces (see Section 5.5).

Unfortunately the concepts of the hyper-surface in line-element geometry have previously been based on several definitions none of which meets the assertion of the natural claim of a relativistic gas theory; i.e. they cannot be connected to the natural generalization of the concept of phase-space volume which is so familiar in the non-relativistic theory. 
One of these definitions is carried out as follows: Let the ensemble of line-elements $\left\{x^{\mu}, v^{\mu}\right\}$ be called a hyper-surface of the line-element space $\mathscr{L}$ the position co-ordinates $\left\{x^{\mu}\right\}$ of which are lying on a three-dimensional hypersurface of four-dimensional co-ordinate space, the directions of which are either perpendicular or tangential to the hyper-surface considered. In terms of another definition the position co-ordinates are lying again on the above mentioned hyper-surface and the angles of inclination of the directions determined by the homogeneous direction co-ordinates $\left\{v^{\mu}\right\}$ are constant in respect of the normal unit vector of the surface.

Neither of these definitions is adequate for our purposes, namely, the hyper-surfaces introduced in this way are $(3+2)$ - and $(3+0)$-dimensional, respectively. Since we are trying to introduce a hyper-surface with $(3+3)$ dimension, this definition of the hyper-surface would recall the familiar definition of the nonrelativistic theory at a given instant of time, i.e. on the hyper-surface $x_{0}=$ const. of the co-ordinate space.

As a matter of fact, we have to suggest a new definition of the hypersurfaces in the framework of general line-element geometry:

As hyper-surface of the geometrized relativistic phase-space the ensemble of the line-elements $\left\{x^{\mu}=x^{\mu}\left(u^{i}\right), p^{\mu}\right\}$ or $\left\{x^{\mu}=x^{\prime \prime}\left(u^{1}\right), \xi_{i}\right\}(i=1,2,3)$ will be denoted where $\left\{u^{i}\right\}$ and $u^{i}=$ const. mean respectively the parameters and the parametric lines of the three-dimensional hyper-surface of the es-ordinates of the line-elements $\left\{x^{u}, p^{i x}\right\}$.

4.1. The invariant volume-element of the co-ordinate-space. In order to define the invariant volume-element of the configuration-space we have to keep in mind that of the familiar scalar density of the Riemannian space-time. To prepare the analytical definition of the suggested general definition of the relativistic phase-space volume-element let the introduction of the well-known Riemannian invariant volume-element be discussed in some detail.

Let the set of quantities

$$
\varepsilon_{\alpha \beta \gamma \delta} \stackrel{\text { des }}{=}\left\{\begin{aligned}
+1, & \text { if } \begin{array}{rl}
\{\alpha, \beta, \gamma, \delta\} \text { means an even permutation } \\
\text { of the numbers }\{0,1,2,3\}
\end{array} \\
-1, & \begin{array}{l}
\text { if }\{\alpha, \beta, \gamma, \delta\} \text { means an odd permutation } \\
\text { of the numbers }\{0,1,2,3\}
\end{array} \\
0, & \begin{array}{l}
\text { if at least two of the indices } \alpha, \beta, \gamma, \delta \\
\text { agree }
\end{array}
\end{aligned}\right.
$$

be introduced which are per definitionem anti-symmetric in all their indices, as well as the pseudo-tensor

$$
\eta_{\alpha \beta \gamma \delta} \stackrel{\text { def }}{=} \sqrt{-g} \varepsilon_{\alpha \beta \gamma \delta} \quad\left(g \stackrel{\text { def }}{=} \operatorname{det}\left|g_{\mu \nu}\right|\right)
$$


with the law of transformation

$$
\eta_{\alpha^{\prime} \beta^{\prime} \gamma^{\prime} \delta^{\prime}}=\operatorname{sgm}\{d\} \frac{\partial x^{\alpha}}{\partial x^{\alpha^{\prime}}} \cdot \frac{\partial x^{\beta}}{\partial x^{\beta^{\prime}}} \frac{\partial x^{\gamma}}{\partial x^{\gamma^{\prime}}} \frac{\partial x^{\delta}}{\partial x^{\delta^{\prime}}} \eta_{a \beta \gamma \delta}
$$

be defined. Then the normal vector to the hyper-surface $x^{\mu}=x^{\mu}\left(u^{i}\right)$ of the configuration-space in the form:

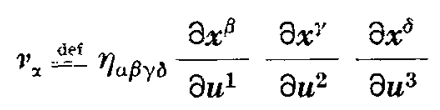

can be obtained, where $\partial x^{\beta} / \partial u^{i}$ denotes the tangents to the parametric lines $\boldsymbol{u}^{i}=$ const. of the surface considered.

The length of the normal vector $v_{\alpha}$ one calculates in a straightforward way:

with

$$
\boldsymbol{v}_{\mathrm{a}} \boldsymbol{v}^{\alpha}=g^{*}
$$

$$
g_{i k}^{*} \stackrel{\text { def }}{=} g_{\alpha \beta} \frac{\partial x^{\alpha}}{\partial u^{i}} \frac{\partial x^{\beta}}{\partial u^{k}} \text { and } g^{*} \stackrel{\text { def }}{\underline{\operatorname{det}}}\left|g_{i k}^{*}\right| \text {. }
$$

This means, however, that the unit normal vector to the hyper-surface

is given by

$$
\left\{x^{\mu}=x^{\mu}\left(u^{1}, u^{2}, u^{3}\right), p^{\mu}\right\}
$$

$$
n_{\alpha}=\frac{1}{\sqrt{\left|g^{*}\right|}} v_{\alpha} \text {. }
$$

Let us suppose in the following that the vectors $v_{x}$ and $n_{\alpha}$ are time-like vectors, i.e. $g^{*}>0$; then the set of tangents to the hyper-surface and the hyper-surface itself will be called space-like.

Keeping this familiar definition in mind, the oriented hyper-surface elements can be introduced in the case of the relativistic configuration-space also by means of the definition

with

$$
d f_{\varrho} \stackrel{\text { def }}{=} v_{\varrho} d u^{1} d u^{2} d u^{3}=n_{\varrho} d f
$$

$$
d f \stackrel{\text { dè̉ }}{=} \sqrt{\left|g^{*}\right|} d u^{1} d u^{3} d u^{3}
$$

being the invariant measure of the hyper-surface element.

Considering the curve $x^{e}=x^{2}(s)$ of the configuration-space $s$ again being the parameter of the length of arc of the line-element geometry, let us suppose that the unit tangent of the curve coincides with the unit normal vector of the hyper-surface in the crossing point of the curve and the hyper-surface

$$
\frac{d x^{e}}{d s}=n^{e} \text { or } d x^{e}==n^{e} d s
$$


then the analytical definition of the invariant volume-element of the configuration. space can in general be given by

$$
d V \stackrel{\text { def }}{=} \frac{\sqrt{-g}}{\sqrt{\left|g^{*}\right|}} d x^{e} d f_{\varrho}=\frac{\sqrt{-g}}{\sqrt{\left|g^{*}\right|}} n^{n} n_{\varrho} d f d s-\frac{\sqrt{-g}}{\sqrt{\left|g^{*}\right|}} d f d s .
$$

In the particular important special case of parametrization

$$
x^{0} \equiv s, x^{i} \equiv u^{i} \quad(i=1,2,3)
$$

the formally well-known formula:

$$
d V=\sqrt{-g} d x^{0} d x^{1} d x^{2} d x^{3} \stackrel{\text { def }}{=} \sqrt{-g} d^{4} x
$$

can be obtained.

4.2. The invariant volume-element of the local momentum-space. Owing to the definition (3.4.3) of the inhomogeneous direction co-ordinates $\left\{\xi_{i}\right\}$, they are invariants of the group of the transformations of the co-ordinates. However, if instead of the line-element $\left\{x^{\mu}, p^{\mu}\right\}$ the line-element $\left\{x^{\mu}, p^{\mu}+D p^{\mu}\right\}$ is considered, the inhomogeneous direction co-ordinates are, of course, changed and their infinitesimal ehanges of first order being linear in the covariant differential of the vectors $p^{t h}$

$$
D p^{\mu}=\left(d p^{z}+\widetilde{\Gamma}_{x \cdot \lambda}^{\tau} p^{\varkappa} d x^{\lambda}\right)\left(\delta_{\tau}^{\mu}+p^{\mu} A_{t}\right) \stackrel{\text { dtf }}{=} \omega^{\mu}(d) \equiv \omega^{\mu}
$$

can be calculated based on eqs. (3.4.3) as follows:

$$
\begin{array}{r}
d \xi_{i}=g_{\mu \nu}\left(x^{\alpha}, a^{\alpha}+D p^{\alpha}\right) \underset{i}{\lambda^{\mu}}\left(p^{\nu}+D p^{\nu}\right) / m_{0}-g_{\mu \nu}\left(\alpha^{\alpha}, p^{\alpha}\right) \underset{i}{\lambda^{\mu}} p^{\nu} / m_{0} \approx \\
\approx\left\{g_{\mu \nu} \lambda_{i}^{\mu} \omega^{\nu}+\frac{\partial g_{\mu \nu}}{\partial p^{\alpha}} \omega^{\alpha} \underset{i}{\lambda^{\mu}}\right\} / m_{0}=\frac{1}{m_{0}}\left\{\omega_{\mu}+2 A_{\mu * a} \omega^{\alpha}\right\} \underset{i}{\lambda^{\mu},}
\end{array}
$$

where the abbreviations introduced in eqs. (3.4.8)-(3.4.10) have been used.

Owing to the obvious invariance of $d \xi_{i}$ against any co-ordinate transformation, the invariant volume-element of the local momentum-space at an arbitrary but fixed point $\left\{x^{\mu}\right\}$ of the configuration-space can, of course, be defined as follows:

$$
d P \stackrel{\text { def }}{=} m_{0}^{3} d \xi_{1} d \xi_{2} d \xi_{3} \stackrel{\text { def }}{=} m_{0}^{3} d^{3} \xi
$$

where the factor $m_{0}^{3}$ has to be introduced in order to maintain the correct physical dimensions of the volume-element of the momentum-space. Although $d P$ is an invariant of the group $\varrho_{x x}$, it will generally change if the internal transformations

$$
\xi_{i^{\prime}}=\xi_{i^{\prime}}\left(\xi_{i}\right)
$$


of the group $Q_{f \xi}$ are considered. Bearing in mind that the transformations $Q_{f \xi}$ are homogeneous linear orthogonal transformations - i.e., they are isomorphic to the three-dimensional subgroup of the Lorentz transformations (3.2.13) - of the type

we have

$$
\xi_{i^{\prime}}=D_{i}^{k} \xi_{k} \quad\left(D \equiv \operatorname{det}\left|D_{i}^{k}\right|= \pm 1\right)
$$

$$
d^{3} \xi^{\prime} \equiv d \xi_{1}^{\prime} d \xi_{2}^{\prime} d \xi_{3}^{\prime}=\frac{\partial\left(\xi_{1}^{\prime}, \xi_{2}^{\prime}, \xi_{3}^{\prime}\right)}{\partial\left(\xi_{1}, \xi_{2}, \xi_{3}\right)} d \xi_{1} d \xi_{2} d \xi_{3}=s g m\{D\} d^{3} \xi
$$

and, as a matter of fact, $d P$ will be a pseudo-scalar under the group $\mathscr{G}_{\xi}$ of the internal transformations. Indeed, the invariant volume-element of the local momentum-space depends on the orientation of the basic $\lambda$-triad.

4.3. The general definition of the pseudo-scalar volume-element of the relativistic phase-space. The underlying general group $\varphi_{f}$ in the background of the concept of the relativistic phase-space - corresponding to the relativistic generalization of the group of the contact transformations of classical dynamics - is the direct product of the groups of the external and internal transformations, i.e.,

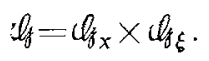

This means, however, that as the relativistic phase-space volume-element in terms of the parametrization (4.1.12) the expression

$$
d \Omega \stackrel{\text { def }}{=} m_{0}^{3} \sqrt{-g} d x^{0} d x^{1} d x^{2} d x^{3} d \xi_{1} d \xi_{2} d \xi_{3}=m_{0}^{3} \sqrt{-g} d^{4} x d^{3} \xi
$$

can be introduced, being a pseudo-scalar of the group $Q_{*}$. The scalar factor $m_{0}^{3}$ is again considered to keep the correct physical dimensions of the phase-space volume-element.

In fact, the relativistic phase-space volume-element may be oriented; let it be denoted as a positive one if the underlying $\lambda$-triad is right-handed.

At a given instant of time, i.e, on the hyper-plane $x_{0}=$ const. of the configuration-space, the phase-space volume-element is reduced to the form

$$
d \Omega_{0} \stackrel{\text { def }}{=} m_{0}^{3} \sqrt{-g} d x^{1} d x^{2} d x^{3} d \xi_{1} d \xi_{2} d \xi_{3}=m_{0}^{3} \sqrt{-g} d^{3} x d^{3} \xi
$$

which is the direct generalization of the well-known expression of non-relativistic gas theory. For the sake of simplicity in the following $d \Omega_{0}$ will be called the momentary expression of the phase-space volume-element $d \Omega$.

Finally, owing to the general definition (3.4.3) of the inhomogeneous direction coordinates $\left\{\xi_{i}\right\}$, let the explicit forms of the relativistic phase-space volume-element be ol,tained in two important cases, in special frames of reference defined in different underlying rnetrical space-times: 
(1) Considering a pseudo-Euclidean space-time continuum with the metrical fundamental tensor (2.0.1), let us first suppose that the axes of the $\lambda^{+}$-triad due to its orientation in the rest frame of reference $K^{0}$ are given by (2.4.6). Then, based on the definitions (4.2.2) of the scalar differentials $d \xi_{i}$ of the inhomogeneous direction co-ordinates, one obtains

$$
d \xi_{i}=-d p^{i} / m_{0}=d p_{i} / m_{0} .
$$

This means, however, that the relativistic phase-space volume-element is given in this case by

$$
d \Omega=d x^{0} d x^{1} d x^{2} d x^{3} d p_{1} d p_{2} d p_{3} \stackrel{\text { def }}{=} d^{4} x d^{3} p_{\mathrm{cov}}
$$

and its momentary expression, i. e. its expression on the $x^{0}=$ const. hyper-plane can be put in the form

$$
d \Omega_{0}=d x^{1} d x^{2} d x^{3} d p_{1} d p_{2} d p_{3} \equiv d^{3} x d^{3} p_{\mathrm{cov}},
$$

the very familiar expression of the non-relativistic phase-space volume-element.

(2) Now, let us assume that the background space-time continuum is Riemannian. In order to obtain the phase-space volume-element and its momentary expression one has to use the framework of the theory of external forms, $[6,15]$. Namely, in terms of the method of the external forms the phase-space volume-element can be written as follows:

$$
d \Omega=m_{0}^{3} \sqrt{-g} d x^{0} \wedge d x^{1} \wedge d x^{2} \wedge d x^{3} \wedge d \xi_{\mathrm{J}} \wedge d \xi_{2} \wedge d \xi_{3}
$$

with the temporarily more favourable abbreviation of the commutators, e. g., $A$ and $B$ :

$$
A \wedge B \stackrel{\text { def }}{=}[A, B] .
$$

With this frame of reference and orientation of the $\lambda^{+}$-triad in mind we have

$$
d \xi_{i}=D_{p_{i}}=\frac{1}{m_{0}}\left(d p_{i}-\left\{\begin{array}{c}
x \\
i \cdot \lambda
\end{array}\right\} p_{k} d x^{\lambda}\right) .
$$

Nevertheless, owing to identity

finally, the expressions

$$
\mathrm{d} x^{\mu} \wedge d x^{y} \equiv 0
$$

and

$$
d \Omega=\sqrt{-g} d^{4} x d^{5} p_{\mathrm{cov}}
$$

can be obtained.

$$
d \Omega_{0}=\sqrt{-g} d^{3} x d^{3} p_{\mathrm{cov}}
$$

Further important particular cases and a re-definition of $d \Omega_{c}$ will be discussed in in detail below, especially in Section 4.5 .

4.4. Generalization of Chernikov's momentary phase-space volume-element. As was mentioned in Section 2.1 - in order to take into account the restricted number of dimensions of the local four-momentum-space - CHernikov had to take an artificial group of transformations as an underlying geometrical background of his relativistic theory of gases. The definition of his transformation group has been suggested by means of the formulae:

$$
x^{\mu \prime}=x^{\mu}\left(x^{\mu}\right), p^{k \prime}=\frac{\partial \alpha^{k \prime}}{\partial x^{\alpha}} p^{\alpha}\left(\Delta+0 ; \mu, \mu^{\prime}, x=0,1,2,3 ; k^{\prime}=1,2,3\right)
$$

keeping the explicit expression (3.0.2) of the time component of the momentum 
in mind. It can easily be proved that the Jacobian of this transformation can be factorized:

$$
\partial \stackrel{\text { dei }}{=} \frac{\partial\left(x^{0^{\prime}}, x^{1^{\prime}}, x^{2^{\prime}}, x^{3^{\prime}}, p^{1^{\prime}}, p^{2^{\prime}}, p^{3^{\prime}}\right)}{\partial\left(x^{0}, x^{1}, x^{2}, x^{3}, p^{1}, p^{2}, p^{3}\right)}=\Delta \frac{\partial\left(p^{1^{\prime}}, p^{2 \prime}, p^{3^{\prime}}\right)}{\partial\left(p^{1}, p^{2}, p^{3}\right)}
$$

Owing to eqs. (4.4.1), (3.4.9), (3.4.10) and the identity

$$
\frac{\partial \boldsymbol{p}^{k \prime}}{\partial \boldsymbol{p}^{r}}=\frac{\partial \boldsymbol{p}^{k \prime}}{\partial \boldsymbol{x}^{\prime}}+\frac{\partial \boldsymbol{x}^{k \prime}}{\partial \boldsymbol{x}^{0}} \frac{\partial \boldsymbol{x}^{0}}{\partial \boldsymbol{p}^{r}}
$$

based on eq. (3.0.2) we have

$$
\frac{\partial p^{0}}{\partial p^{r}}=-\frac{p+A_{* *} r}{p_{0}+A_{* * 0}}
$$

consequently, for the Jacobian the final expression

can be obtained.

$$
\mathscr{Z}=\frac{p_{0}^{\prime}-A_{* * 0}^{\prime}}{p_{0}+A_{* * 0}} \Delta^{2}
$$

In fact, the analytic expression of the relativistic phase-space volumeelement in terms of the external forms - analogously to eq. (4.3.7) - in the form

$$
d \widetilde{\Omega} \stackrel{\text { def }}{=} \frac{-g}{p_{0}+A_{* * 0}} d x_{0} \wedge d x^{1} \wedge d x^{2} \wedge d x^{3} \wedge D p^{1} \wedge D p^{2} \wedge D p^{3}
$$

has to be introduced. Owing to the definition (4.2.1) of the covariant differentials $D p^{\alpha}$, this expression of $d \widetilde{\Omega}$, owing to the identity (4.3.10), may also be put in the form

$$
d \widetilde{\Omega}=\frac{-g}{p_{0}+A_{* * 0}} d^{4} x d^{3} p_{\text {contr }}\left(d^{3} p_{\text {contr }} \stackrel{\text { dep }}{=} d p^{1} d p^{2} d p^{3}\right)
$$

The adequate expression of the momentary phase-space volume-element is given by

$$
d \widetilde{\Omega}_{0}=\frac{-g}{p_{0}+A_{* * 0}} d^{3} x d^{3} p_{\text {contr }}
$$

In the case of gaseous systems without intrinsic dynamic anisotropy - i.e. if in terms of the suggested geometrized model of the relativistic phasespace the underlying background line-element space is reduced to a Riemannian 
one - the metrical fundamental tensor of the field does not depend on the homogeneous direction co-ordinates. Consequently, the torsion tensor defined by eq. (3.4.9) vanishes. Of course, also the contracted torsion tensor, $A_{* * k}=$ $=0$; i.e. eqs. (4.4.6) and (4.4.7) are reduced to

and

$$
d \widetilde{\Omega}_{R}=-\frac{g}{p_{0}} d^{4} x d^{3} p_{\text {contr }}
$$

$$
d \widetilde{\Omega}_{R 0}=-\frac{g}{p_{0}} d^{3} x d^{3} p_{\text {contr }}
$$

respectively, being precisely the formulae first obtained in this special case by Chernikov [6]. One sees immediately also that these volume-elements are pseudo-scalars depending on the sign of $p^{0}$. By means of the suggested generalization in eqs. (4.4.6) and (4.4.7), respectively, the sign of $p_{0}-$ in this connection - has to be replaced by that of $p_{0}+A_{* * 0^{*}}$

We shall see in Section 4.6. that the definition (4.3.3) of the momentary phase-space volume-element in the language of inhomogeneous direction coordinates is much more favourable for practical calculations than the present formalism based on the group of the Chernikov's transformations because then warning and seemingly insurmountable difficulties of a pure technical character appear when carrying out some important applications.

4.5. Liouville's Relativistic Theorem. In order to explain the physical meaning of $d \Omega_{0}$, i.e. to interpret the momentary expression of the phase-space volume-element in more familiar language and to investigate its invariant properties it seems to be worth-while to improve the above, pure geometrical definition of the relativistic phase-space volume-element in terms of phasespace formalism. Such an approach was most recently suggested by LiNDQUIST [36] - having SASAKI's tangent-bundle geometry in mind [37] published shortly after the appearance of [35] and we shall see that both definitions of $d \Omega_{0}$ are equivalent.

Let us start with the definition (4.1.4) of the unit normal vector $n_{\alpha}$ to the $\sigma$ hyper-surface $x^{\lambda}=x^{\lambda}\left(u^{j}\right)$ and denote the tangents to the parametric lines $u^{j}=$ const. of the surface considered by $d_{j} x^{\lambda} \sim d x^{\lambda} / d u^{j}(j=1,2,3)$. We may emphasize again that $n_{\alpha}$ is now an arbitrary time-like unit vector, being orthogonal to the space-like hyper-surface spanned by the vectors $d_{j} x^{\lambda}$, i.e.

where

$$
\sqrt{-g} \varepsilon_{\lambda a \beta ;}, d_{1} x^{\alpha} d_{2} x^{\beta} d_{3} x^{\gamma}=d \mathscr{O}^{(\sigma)} n_{\lambda}
$$

$$
d \mathscr{P}^{\mathscr{O}_{\sigma}} \stackrel{\text { def }}{=} \sqrt{-g} \varepsilon_{\lambda \alpha \beta \gamma} n^{\lambda} d_{1} x^{\alpha} d_{2} x^{\beta} d_{4} x^{\gamma}
$$

means a constant of proportionality being an invariant volume-element orthogonal to $n_{\lambda}$. Keeping our eqs. (4.1.5)-(4.1.9) in mind, its geometrical meaning can immediately be understood as follows. 
To begin, let us remember that at this point we have supposed in Section 4.1. that the unit tangent of the world-line $x^{\lambda}=x^{\lambda}(\tau)$ of the gaseous particles coincides with the unit normal vector $n_{\alpha}$ in the crossing point of the curve and the hyper-surface and the invariant volume-element of the configuration-space has been defined in this special case. Now, let us generalize our method to a certain extent by supposing that on the one hand the unit normals $n_{\alpha}$ of the hyper-surfaces are defined along a world-line by some kind of parallel transports - e.g. by FERMI-WALKER transport - and on the other hand that the solid angle between the tangents of the world-line

$$
\frac{d x^{\lambda}}{d \tau}=p^{\lambda} / m_{0}, \quad d x^{\lambda}=m_{0}^{-1} p^{\lambda} d \tau
$$

and $n_{\alpha}$ is constant, where $\mathrm{d} \tau=d s$ means the proper time-difference which, in our system of units, is equal to $d s$, i.e. to the infinitesimal change of length of arc along the world-line. This means, however, that

$$
\begin{aligned}
d V^{(\sigma)} \stackrel{\text { def }}{=} m_{0}^{-1}\left(p^{\lambda} n_{\lambda}\right) d \mathscr{V}^{\mathscr{( \sigma )}} d \tau & \equiv m_{0 \lambda}^{-1} \sqrt{-g} \varepsilon_{\lambda(\alpha \gamma \gamma} p^{\lambda} d_{1} x^{\alpha} d_{2} x^{\beta} d_{3} x^{\gamma} \equiv \\
& \equiv m_{0}^{-1}\left(p_{\lambda} n^{\lambda}\right) d \mathscr{V}^{(\sigma)} d \tau
\end{aligned}
$$

is exactly the four-dimensional invariant volume-element of the configurationspace.

In the particular case when $x^{\lambda}=x^{\lambda}(\tau)$ is the orthogonal trajectory of our $\sigma$ hyper-surface - i.e. $p_{\lambda}$ and $n^{\prime}$ are parallel $-m_{v}^{-1}\left(p_{\lambda} n^{\lambda}\right)=1$ and we reproduce our previous result: $d V^{(\sigma)}=d \mathscr{V}^{(\sigma)} d \tau=d V$. Indeed, if in the rest frame of reference $K^{0}$ of the gaseous system, as in the case of eq. (4.1.12), the parametrization

were to be introduced,

$$
x^{0}=\tau, \quad x^{j}=u^{j} \quad(j=1,2,3)
$$

$$
d_{1} x^{\alpha}=\left\{0, d x^{1}, 0,0\right\}, d_{2} x^{\beta}=\left\{0,0, d x^{2}, 0\right\}, d_{3} x^{\gamma}=\left\{0,0,0, d x^{3}\right\}
$$

and

$$
d V^{(\sigma)}=d \mathscr{V} d \tau=\sqrt{-g} d^{4} x
$$

can be obtained, in full agreement with eq. (4.1.13).

As matters stand, it seems to be worth-while to define the invariant quantity

$$
d V_{0}^{(\sigma)} \stackrel{\text { def }}{=} \frac{d V^{(\sigma)}}{d \tau} \equiv m_{0}^{-1}\left(p_{\lambda} n^{\lambda}\right) d \mathscr{V}^{(\sigma)}
$$

which may be interpreted as the invariant three-dimensional volume-element pro unit of the length of arc along the world-line $x^{\lambda}=x^{\lambda}(\tau)$. Of course, in the 
particular case discussed above, when $p_{\hat{\lambda}}$ is parallel to $n^{\lambda}$, the expression of $d V_{0}^{(\sigma)}$ is reduced to $d \mathscr{V}^{\left(\sigma_{0}\right)} \equiv d V_{0} \equiv d^{3} x$.

It would be a mere repetition of our previous discussions if we were to follow the familiar way of thinking to construct the phase-space volumeelement in general terms. Therefore, without any considerable loss of generality, we may suppose that the space-time background of our theory is a stationary four-dimensional Riemannian manifold with the metrical fundamental tensor:

$g_{00}=g_{00}(r), g_{11}=-g_{11}(r), g_{22}=-g_{22}(r), g_{33}=-g_{33}(r, \vartheta) ; g_{\mu v}=0(\mu \neq v)$,

where $\{r, \vartheta, \varphi\}$ are polar co-ordinates in the three-dimensional space-like subspace. In fact, $g^{\prime \mu}=g_{\mu \mu}^{-1}[$ no summation over $\mu]$ and due to the normalization conditions

$$
g_{\mu \nu} n^{\mu} n^{\nu}=1 \text { and } g^{\mu \nu} p_{\mu} p_{\nu}=m_{0}^{2},
$$

for the unit normal vector and for the four-momentum in the rest frame of reference $K^{0}$, the rather explicit expressions

$$
n^{\lambda}=\left\{1 / \sqrt{g_{00}}, 0,0,0\right\} \text { and } p_{\lambda}=\left\{m_{0} / \sqrt{g^{00}}, 0,0,0\right\}
$$

can be obtained.

Having the stationary Riemannian manifold with the metrical fundamental tensor (4.5.9) in mind, in order to introduce the inhomogeneous direction co-ordinates one has to refer back to their original definition

$$
\xi_{j}=\frac{j_{j}^{\lambda^{\mu}} \boldsymbol{p}_{\mu}}{\sqrt{g_{\alpha \beta} \lambda_{j}^{\alpha} \lambda_{j}^{\beta}} \sqrt{g^{g^{\sigma \alpha}} p_{\varrho} p_{\sigma}}} \quad(j=1,2,3) .
$$

We may suppose that $\lambda^{\mu} \sim d_{j} x^{\mu}$, then, if we use the particularly defined $\lambda^{+}$-triad $(2.4 .6)$ in $K^{0}$ we get

$$
\xi_{j}=\left(m_{0} \sqrt{g_{j j}}\right)^{-1} p_{j} \quad(j=1,2,3)
$$

It must be constantly borne in mind that all $\xi_{j^{-S}}$ are invariants of the coordinate transformations, meaning their values are unchanged in other frames of references, too.

Owing to our previous general result (4.3.6), the volume-element of the momentum-space can be written in the form

$$
d P \equiv m_{0}^{3} d^{3} \xi=m_{0}^{3} \xi^{2} \sin \Theta d \xi d \Theta d \Phi
$$


where $\{\xi, \theta, \Phi\}$ are polar co-ordinates in the momentum-space with

$$
\xi^{2} \stackrel{\text { def }}{=} \xi_{1}^{2}+\xi_{2}^{2}+\xi_{3}^{2}=m_{0}^{-2}\left\{g^{11} p_{1}^{2}+g^{22} p_{2}^{2}+g^{33} p_{3}^{2}\right\}
$$

The triad- and tetrad-axes are usually defined with their contravariant components. This is why in the definition of the inhomogeneous direction coordinates and on the occasion of the normalization, the covariant components of the four-momenta were introduced. As a consequence, it seems to be natural to represent the energy of the gaseous particle in terms of the covariant timelike component of the four-momentum:

i.e.

$$
p_{0} \equiv \varepsilon
$$

$$
g^{00} p_{0}^{2}-\left\{g^{11} p_{1}^{2}+g^{22} p_{2}^{2}+g^{33} p_{3}^{2}\right\} \equiv g^{00} p_{0}^{2}-p^{2}=g^{00} \varepsilon^{2}-m_{0}^{2} \xi^{2}=m_{0}^{2}
$$

with

Considering that

$$
p \stackrel{\text { def }}{=}\left\{g^{11} p_{1}^{2}+g^{22} p_{2}^{2}+g^{33} p_{9}^{2}\right\}^{1 / 2}
$$

$$
\begin{gathered}
\xi=m_{0}^{-1} p=\left\{\frac{\varepsilon^{2}}{m_{0}^{2}} g^{00}-1\right\}^{1 / 2} ; d \xi=m_{0}^{-2} g^{00}\left\{\frac{\varepsilon^{2}}{m_{0}^{2}}-1\right\}^{-1 / 2} ; \\
d \omega \equiv \sin \Theta d \Theta d \Phi,
\end{gathered}
$$

for the volume-element of the momentum-space the co-ordinate invariant expression

$$
d P=m_{0}^{3} \xi^{2} d \xi d \omega=m_{0} g^{06}\left\{\frac{\varepsilon^{2}}{m_{0}^{2}} g^{00}-1\right\}^{1 / 2} \varepsilon d \varepsilon d \omega \equiv g^{00} \varepsilon p d \varepsilon d \omega
$$

can be obtained.

Finally, from eq. (4.3.2) the invariant volume-element of the $(4+3)$ dimensional phase-space has the form

$$
d Q^{(\sigma)}=d V^{(\sigma)} d P=g^{00}\left(p_{\lambda} n^{\lambda}\right)\left\{\frac{\varepsilon^{2}}{m_{0}^{2}} g^{00}-1\right\}^{1 / 2} \varepsilon d \varepsilon d \omega d \mathscr{V}^{\mu(\sigma)} d \tau
$$

and the invariant $(3+3)$-dimensional volume-element on the hyper-surface pro the unit length of arc along the world-line can be written as

$$
\begin{aligned}
d Q_{0}^{(\sigma)} \stackrel{\text { def }}{=} \frac{d \Omega_{0}^{(\sigma)}}{d \tau} & =g^{00}\left(p_{\lambda} n^{\lambda}\right)\left\{\frac{\varepsilon^{2}}{m_{0}^{2}} g^{00}-1\right\}^{1 / 2} \varepsilon d \varepsilon d \omega d \mathscr{V}^{\mathscr{(}(\sigma)}= \\
& =m_{0}^{-1} g^{00}\left(p_{\lambda} n^{\lambda}\right) \varepsilon p d \varepsilon d \omega d \mathscr{V}^{(\sigma)}
\end{aligned}
$$


which is equivalent to the momentary value of phase-space volume-element introduced in eqs. (4.3.3) and (4.3.12) on the hyper-plane $x^{0}=$ const.

In particular, in the case of parallel $p_{\lambda}$ and $n^{\lambda}$ one gets

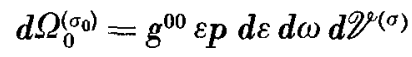

and in the rest frame of reference $K^{0}$, if pseudo-Euclidean metric were to be introduced locally, the familiar non-relativistic phase-space volume-element could be obtained:

$$
d \Omega_{0}^{(l p E)}=\varepsilon p d \varepsilon d \omega d \mathscr{V}^{\circ}\left(\sigma_{0}\right)=\varepsilon p d^{3} x d \varepsilon d \omega
$$

The phase-space volume-elements $d \Omega$ and $d \Omega_{0}$ as well were invariants of the transformation group $\theta_{x}$ of the configuration-space. In Section 4.3. we mentioned that owing to the possibility of internal inversion in respect to the origin of the $\lambda^{+}$-triad in the case of the general transformation group $\varphi_{k}=\theta_{\delta} \times \varphi_{\xi \xi}$ of the relativistic phase-space, they are pseudo-scalars. Nevertheless, keeping the definition (4.5.1) of $n_{\lambda}$ in mind, as a result of the parallelism between $d_{j} x^{\mu}$ and the triad-axes $\lambda^{\mu}(j=1,2,3)$ and of the appearance of the factor $\left(p_{\lambda} n^{2}\right)$, our new volume-elements $d \Omega^{(\sigma)}$ and $d \Omega_{0}^{(\sigma)}$, respectively, are invariants of the general group $Q_{0}=Q_{x} x Q_{\xi \xi}$, too.

The reason why in terms of the relativistic phase-space formalism as momentary value of the phase-space volume-element exactly the familiar expression of the non-relativistic phase-space volume-element was obtained can be found in the fact that the suggested hyper-geometrization is a natural geometrization of the real space-time and dynamic relations. What is more, if the "natural motion of the phase-space" as FERMI-WALKER transport or "geodesic flow" along the world-line of the gaseous particles were to be interpreted, $d \Omega_{0}^{(\sigma)}$ is an invariant along the world-line of the gaseous particles. Having this important property of $d \Omega_{0}^{(\sigma)}$ in mind, if a bundle of trajectories, i.e., a "phase-tube" is considered generated by a section $d \Omega_{0}^{(\sigma)}$ of the world lines of neighbouring phase-points, the relativistic form of Liouville's Theorem can be formulated in the following way:

$$
\int_{\Omega_{0}^{(\sigma)}} d \Omega_{0}^{(\sigma)}=\int_{\Omega_{0}^{(\sigma)}} d \Omega_{0}^{(\sigma) \prime}
$$

where $\Omega_{0}^{(\sigma)^{\prime}}$ is the image of $\Omega_{0}^{(\sigma)}$ via the natural motion, i.e. by the geodesic flow of the phase-space.

4.6. The zero-point kinetic energy of perfect fermion gases. In addition to the natural theoretical interest in the generalization of important physical concepts, investigations to deal with the definitions of the energy and momentum of particles on the Fermi level in the case of relativistic fermion gases in Riemannian space-time continuums are also suggested by problems more recently raised in neutrino-astronomy [38-4.1]. 
Considering a completely degenerate fermion gas, i. e., - - for the sake of simplicity a perfect system of fermions at zero point of absolute temperature, the fermions are distributed over the different quantum states in such a way that the total energy of the gas has its smallest possible value. Owing to the fundamental feature of fermion gases, - i. e. to Pauli's exclusion principle - no more than one fermion may be in any one state, the fermions fill all states with energies between the smallest (equal to zero) and some largest value wich is determined by the number $N$ of the fermions. Let us suppose that the fermion gas is in a volume $\mathscr{V}$ of the configuration space, then the smallest possible value of its total energy - the so-called zero point kinetic energy $-E_{0}$ can essentially be calculated based on the framework of the non-relativistic phase-space method if an adequate definition of the phase-space volumeelement is used.

For the sake of simplicity let the relativistic neutrino gas be considered in Riemannian spaces with spherical symmetry. First the somewhat more general case of fermions with nonvanishing rest mass $\left(m_{0} \neq 0\right)$ will be discussed. Then the results will be specialized for the neutrino gas by the limiting process $m_{0} \rightarrow 0$.

According to the classical results the Fermi momentum and energy of the gaseous particles depend on the mean density $\bar{\varrho}=N / \mathscr{Q}$ of fermions in the configuration-space. This mean density can naturally be defined either in a stationary Riemannian space-time if the universe is closed or in open universes having in mind the framework of infinite systems of fermions, i. e. the limit $N \rightarrow \infty$ and $\not \mathscr{P}^{\rightarrow} \rightarrow \infty$ with the restriction $\bar{\varrho}=$ const. Both cases will be discussed in Riemannian space-time continuums with particularly interesting metrical structures.

Owing to the spherical structure of the space-time continuum and to the dynamic isotropy of systems of the considered particles on the $x^{0}=$ const. hyper-plane the spherical polar co-ordinates $\{r, \theta, \varphi\}$ and $\{p, \theta, \Phi\}$ will, of course, be introduced in the configurationand local momentum-spaces, respectively. In the particular cases to be taken into consideration in the following the components of the metrical fundamental tensor can generally be given as follows:

$$
g_{00}=-h_{0}(r), g_{11}=h_{1}(r), g_{22}=h_{2}(r), g_{33}=h_{3}(r) \sin ^{2} \vartheta ; g_{\mu \nu}=0(\mu \neq v) .
$$

The reason why the signature $(-+++)$ is introduced here instead of the usual one, is that the hyper-sufrace $x^{0}=$ const. is space-like. This means, however, that

Furthermore,

$$
g=-h_{0} h_{1} h_{2} h_{3} \sin ^{2} \theta \text { and } \sqrt{-\bar{g}}=\left\{h_{0} h_{1} h_{2} h_{3}\right\}^{1 / 2} \sin \theta \text {. }
$$

$$
g^{00}=-h_{\theta}^{-1}(r), g^{11}=h_{1}^{-1}(r), g^{22}=h_{2}^{-1}(r), g^{33}=h_{3}^{-1}(r) \sin ^{-2} \vartheta ; g^{\mu v}=0(\mu \neq v) .
$$

Therefore, the normalization condition can be put into the form:

$$
-h_{0}^{-1} p_{0}^{2}+\left\{h_{1}^{-1} p_{1}^{2}+h_{2}^{-1} p_{2}^{2}+h_{3}^{-1} \sin ^{-2} \vartheta p_{3}^{2}\right\}=-m_{0}^{2}
$$

and by using eqs. (4.5.19) we have

$$
\xi=m_{0}^{-1} p=\left\{\frac{\varepsilon^{2}}{m_{0}^{2}} h_{0}^{-1}(r)-1\right\}^{t / 2} .
$$

Finally, the momentary relativistic phase-space volume on the $x^{0}=$ const. hyper plane can be written as follows:

$$
\begin{gathered}
\Omega_{0}=m_{0}^{3} \int_{\alpha}^{R} d x \int_{0}^{\pi} d \vartheta \int_{0}^{2 \pi} d \varphi \int_{0}^{\xi_{F}} d \xi \int_{0}^{\pi} d \Theta \int_{0}^{2 \pi} d \Phi\left\{h_{0} h_{1} h_{2} h_{3}\right\}^{1 / 2} \sin \vartheta \xi^{2} \sin \Theta= \\
=\frac{(4 \pi)^{2}}{3} m_{0}^{3} \int_{0}^{R} d r\left\{h_{0} h_{1} h_{2} h_{3}\right\}^{1 / 2}\left\{\frac{\varepsilon_{f}^{2}}{m_{0}^{2}} h_{0}^{-1}(r)-1\right\}^{3 / 2},
\end{gathered}
$$

where $\xi_{F}$ and $\varepsilon_{F}$ mean the highest possible values of $\xi$ and $\varepsilon$, respectively, on the Fermi level of the momentum-space. The upper limit $R$ of the configuration-space integral means either 
the radius of the universe or in the case of open universes the radius of the spherical symmetrical space-time domain considering before the limiting process $R=\left(3 \mathscr{V}^{\mathscr{T}} / 4 \pi\right)^{1^{\prime 3}} \rightarrow \infty$. The lower limit $\alpha$ is determined by the metrical properties.

In fact, the number of states in the momentum interval $\left(o, p_{F}\right)$ filled with phase-points of the particles is given by $N$ and owing to the definition of the three-dimensional volume in the configuration-space, we have

$$
\mathscr{V}^{\prime}=4 \pi \int_{\alpha}^{R} d r\left\{h_{0} h_{1} h_{2} h_{3}\right\}^{1^{\prime 2}}
$$

and one can write

$$
N=\gamma \frac{(4 \pi)^{2}}{3}\left(\frac{m_{0}}{2 \pi \hbar}\right)^{a} \int_{\alpha}^{R} d r\left\{h_{0} h_{1} h_{2} h_{3}\right\}^{1 / 2}\left\{\frac{\varepsilon_{F}^{2}}{m_{0}^{2}} h_{0}^{-1}(r)-1\right\}^{3 / 2}
$$

where $\gamma$ denotes the number of the internal degrees of freedom in each quantum state (having usually only spin degeneracy in mind). $2 \pi \hbar$ denotes Planck's constant.

Let the mean density of the fermion-gas be defined by

$$
\bar{\varrho}=N / \mathscr{Q},
$$

then its density $\varrho(r)$ on the hyper-plane $x^{0}=$ const. of the configuration-space may be defined by

$$
\bar{\varrho}=\frac{\gamma}{\mathscr{V}} \frac{(4 \pi)^{2}}{3}\left(\frac{m_{0}}{2 \pi \hbar}\right)^{3} \int_{\alpha}^{R} d r\left\{h_{0} h_{1} h_{2} h_{3}\right\}^{1 / 2}\left\{\frac{\varepsilon_{F}^{2}}{m_{0}^{2}} h_{0}^{-1}(r)-1\right\}^{3 / 2}=4 \pi \int_{\alpha}^{R} d r r^{2} \varrho(r) .
$$

Indeed, let the three-dimenional density $\varrho(r)$ be introduced by

$$
\varrho(r) \stackrel{\text { def }}{-} \frac{\gamma}{\mathscr{V}^{2}} \frac{4 \pi}{3}\left(\frac{m_{0}}{\hbar 2 \pi}\right)^{3} \frac{1}{r^{2}}\left\{{ }_{0} h_{1} h_{2} h_{3}\right\}^{1 / 2}\left\{\frac{\varepsilon_{F}^{2}}{\mid m_{0}^{2}} h_{0}^{-1}(r)-1\right\}^{3 / 2} .
$$

So far the Fermi energy of the particles is unknown and in terms of $\bar{\varrho}$ is only implicitly determined by eq. (4.6.10). In order to calculate it explicitly one has to carry out the $r$-integration in eq. $(4,6.10)$.

Finally, it seems to be worth-while to introduce the three-dimensional energy density of the fermion gas, being the corresponding $T_{0}{ }^{\circ}(r)$ component of the energy-momentum tensor of the system. Owing to the definition of the zero point kinetic energy this may be carried out by means of the relation:

$$
E_{0}=\int_{\alpha}^{R} d r \int_{0}^{\pi} d \vartheta \int_{0}^{2 \pi} d \varphi \int_{0}^{\epsilon_{F}} d \Sigma r^{2} \varrho(r) \varepsilon(r)=2 \pi \varepsilon_{F}^{2} \int_{x}^{R} d r r^{2} \varrho(r) \stackrel{\text { def }}{\underline{\underline{y}}}-4 \pi \int_{a}^{R} d r\left\{h_{0} h_{1} h_{2} h_{3}\right\}^{1 / 2} T_{0}^{0}(r),
$$

based on which the definition

$$
T_{0}^{0}(r) \stackrel{\text { def }}{=}-\frac{2 \pi}{3} \frac{\gamma^{\gamma}}{\mathscr{V}^{2}}\left(\frac{m_{0}}{2 \pi \hbar}\right)^{3} \varepsilon_{F}^{2}\left\{\frac{\varepsilon_{F}^{2}}{m_{0}^{3}} h_{0}^{-1}(r)-1\right\}^{3 / 2}
$$

can be suggested.

We are particularly interested in the special case of a relativistic neutrino gas. This means that we have to earry out the limiting process $m_{0} \rightarrow 0$ in the formulae obtained above.

First of all, one observes that owing to eqs. (4.6.9) and (4.6.10), and also, to the fact that owing to the spin degeneracy in the case of neutrinos $\gamma=2$, the mean density $\bar{\varrho}$ of the neutrino gas on the hyper-plane $x^{0}=$ const. of the configuration-space is determined in terms of

$$
\bar{\varrho}=\frac{4 \varepsilon_{F}}{3 \pi \mathscr{V}^{2}} \int_{x}^{R} d r h_{0}^{-1}(r)\left\{h_{1} h_{2} h_{3}\right\}^{1 / 2}
$$




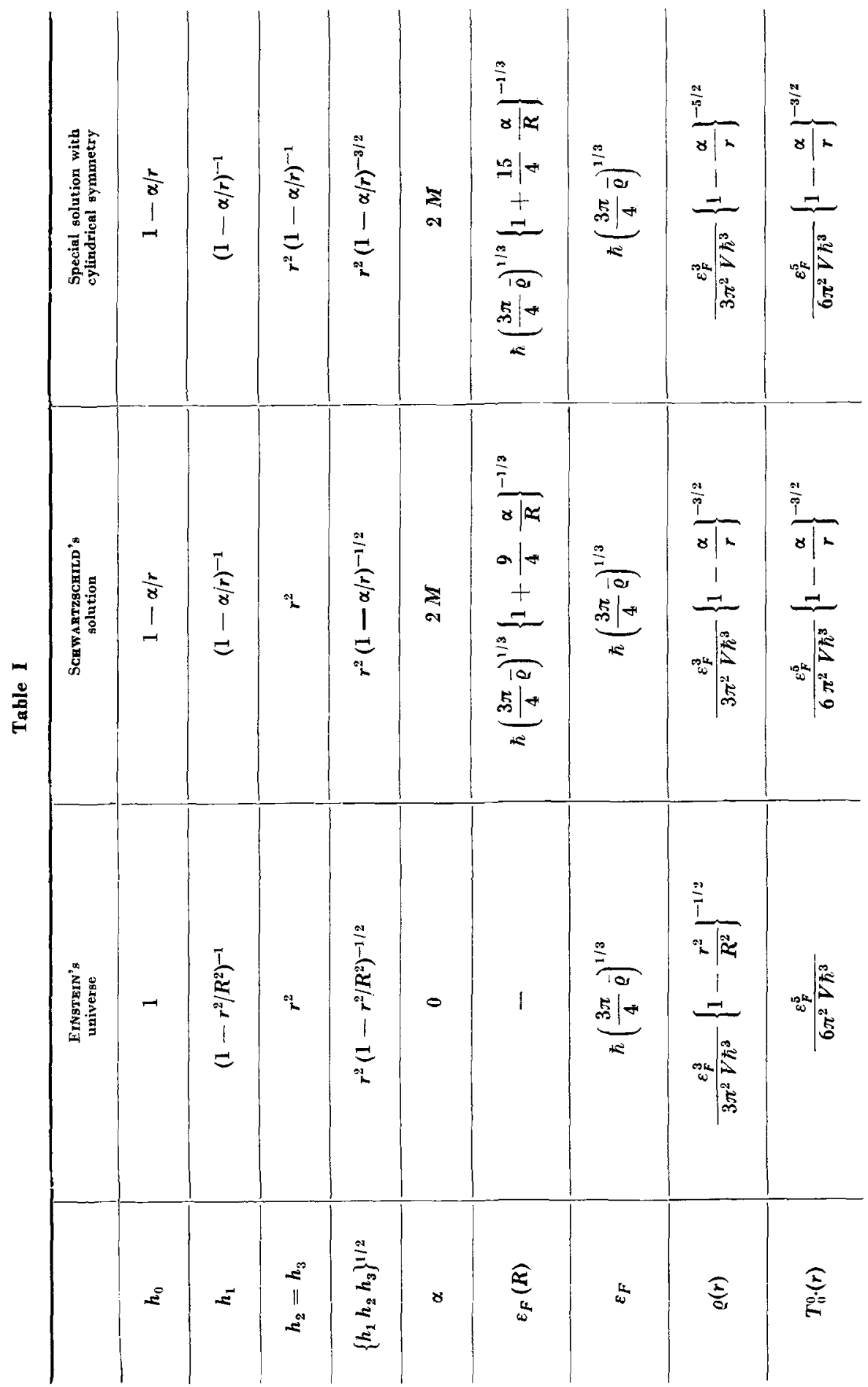


e. th: Fermi energy is given by means of $\bar{Q}$ as

$$
\varepsilon_{F}=\hbar\left[\frac{3 \pi}{4} \bar{\varrho} \int_{\alpha}^{R} d r\left\{h_{0} h_{1} h_{2} h_{3}\right\}^{1 / 2}\right]^{1 / 3}\left[\int_{\alpha}^{R} d r h_{0}^{-1}\left\{h_{1} h_{2} h_{3}\right\}^{1 / 2}\right]^{-1 / 3} .
$$

As to the three-dimensional density (4.6.11) of the neutrino gas and its three-dimensional energy density (4.6.13) on the hyper-plane $x^{0}=$ const. of the configuration-space we have:

and

$$
Q(r)=\frac{\varepsilon_{F}^{3}}{3 \pi^{2}} \frac{1}{\mathscr{V}^{2} \hbar^{3}} \frac{1}{r^{2} h_{0}(r)}\left\{h_{1} h_{2} h_{3}\right\}^{1 / 2}
$$

$$
T_{0}^{0}(r)=-\frac{\varepsilon_{F}^{5}}{6 \pi^{2} \mathscr{V}^{q} \hbar^{3}} h_{0}^{-3 / 2}(r) .
$$

In order to obtain the final results in the case of different Riemannian space-time continuums the explicit expressions of the metrical fundamental tensor in eqs. (4.6.1) have to be taken into account. These are summarized in Table $I$.

I am very grateful to Professor H. Fröhlich for many helpful discussions and for his kind interest in these investigations. I am also indebted to Professor G. Marx for his stimulating criticism concerning the problems connected with the definitions of the phase-space volume and with the interpretation of the distribution functions.

\section{REFERENCES}

28. J. I. Honvâth, Acta Phys. Hung., 24, 205, 1968.

29. P. Nelli, Rend. Acc. Linc. (6), 13, 669, 1931.

30. A. Moón, Acta Sc. Math. Szeged, 17, 85, 1956.

31. J. I. Honváth, Nuovo Cimento (10), 7, 636, 1958.

32. J. L. Synge, Relativity: the General Theory (North-Holland PC., Amsterdam, 1960.)

33. E. Fenmi, Rend. Acc. Linc. (1) 31, 21, 51, 1922.

34. A. G. Walker, Proc. Roy. Soc. Edinburgh, 52, 345, 1932.

35. J. I. Honvâth, Report at the International Anniversary Conference of Relativity (organized by the F. Schiller University), Georgenthal, on the 15-27th February 1965; Wiss. Zs. F. Schiller Univ., 15, $149,1966$.

36. R. W. Lindeuist, Ann. Phys., 37, 487, 1966.

37. S. SASAKI, Tohoku Math. Journ., 10, 338, 1958; 14, 146, 1962.

38. S. Weinberc, Nouvo Cimento, 25, 15, 1962.

39. L. Fodor, Zs. Kövesy and G. Marx, Acta Phys. Hung., 17, 171, 1964.

40. G. MARX, ITP Budapest Report No. 218, 1966.

41. J. I. Horváte, Acta Phys. et Chem. Szeged, 13, 3, 1967. 


\section{О СВЕРХ-ГЕОМЕТРИЗАЦИИ РЕЛЯТИВИСТСКОГО ФАЗОВО- ПРОСТРАНСТВЕННОГО ФОРМАЛИЗМА II}

\section{Я. и. XOPBAT}

\section{P e 3 to me}

В первой части данной рабооты подчеркнвается, что в рамках линейных элементов геометрип с помощью зависимости динамических величин от направлений в пространстве времени возможна геометризация внутренних динамических соотношений рассмотренной системы. Для простоты данная идея сверх-геометризации дискутировалась в случае псевдо-Эвклидового пространственно-временного континуума. В настояџей части, с одной стороны, в рамках так называемого четырехчленного формализма геометрии Риманна и основываясь на непосредственном обоб̈щении предложенного трехчленного формализма с другой, внутренние динамические степени свободы, их симметрии и пространственновременная картина истолкуются и расширяются для неоднородных и анизотропных динамических систем. Наконец, днскутируются одно новое об̆цее определение релятивистического пространственно-временного объема и его связи с предыдуцими предложениями с некоторыми применениями в теории фермионных газов. 